\title{
Sobredosis accidental de enoxaparina en un recién nacido
}

\author{
Enoxaparin overdose in a newborn
}

\author{
Prof. Dr. Miguel Á. Marín Gabriel ${ }^{a, b}$, Lic. Roberto Ortiz Movilla ${ }^{a}$, Lic. Carmen Muñoz Labián ${ }^{a}$, \\ Lic. Miguel F. Sánchez Mateos ${ }^{a}$ y Lic. Rebeca Sánchez Salmador ${ }^{a}$
}

\section{RESUMEN}

La enoxaparina es una heparina de bajo peso molecular utilizada en el período neonatal. Requiere menor monitoreo que la heparina estándar o no fraccionada, si bien es escaso el conocimiento actual acerca de su dosis y de los niveles terapéuticos en los neonatos. Además, existe una información muy limitada respecto del manejo de su sobredosificación en este grupo de edad. Se presenta el primer caso publicado en castellano de un neonato que recibió una dosis de enoxaparina diez veces superior a la terapéutica de forma accidental y en el que se administró una dosis aislada de protamina para revertir su efecto.

Palabras clave: enoxaparina, errores de medicación, protaminas, recién nacido.

\begin{abstract}
Enoxaparin is a low molecular weight heparin used in the neonatal period. It requires less monitoring than standard or unfractionated heparin, although current knowledge about its dose and therapeutic levels in neonates is scarce. In addition, there is very limited information about the management of overdose in this age group. We present the first case published in Spanish of a neonate who accidentally received a dose of enoxaparin ten times higher than the therapeutic one and an isolated dose of protamine to reverse its effect.

Key words: enoxaparin, medication errors, protamines, infant newborn.
\end{abstract}

http: / / dx.doi.org/10.5546/aap.2018.e762

Cómo citar: Marín Gabriel MA, Ortiz Movilla R, Muñoz Labián C, Sánchez Mateos MF, et al. Sobredosis accidental de enoxaparina en un recién nacido. Arch Argent Pediatr 2018;116(6):e762-e764.

a. Departamento de Pediatría, Unidad Neonatal, Hospital Universitario Puerta de Hierro Majadahonda.

b. Departamento de Pediatría, Universidad Autónoma de Madrid, España.

Correspondencia:

Dr. Miguel Á. Marín Gabriel:

mangel.maringa@salud.madrid.org

Financiamiento: Ninguno.

Conflicto de intereses: Ninguno que declarar.

Recibido: 10-2-2018

Aceptado: 30-7-2018

\section{INTRODUCCIÓN}

La enoxaparina es una heparina de bajo peso molecular (HBPM) utilizada en el período neonatal, debido al uso, cada vez más frecuente en recién nacidos, de procedimientos invasivos que implican un aumento del riesgo de fenómenos tromboembólicos. ${ }^{1}$ Por su administración subcutánea y sus características farmacocinéticas, requiere menor monitoreo que la heparina estándar o no fraccionada, si bien es escaso el conocimiento actual acerca de su dosis y de los niveles terapéuticos en los neonatos. ${ }^{2}$ Además, existe una información muy limitada respecto del manejo de su sobredosificación en este grupo de edad, que es especialmente vulnerable a los errores en la administración de medicamentos. ${ }^{3,4}$ Este tipo de errores son mucho más frecuentes durante este período, sobre todo, en los pacientes ingresados en las unidades de cuidados intensivos, y existe menor notificación de estos cuando tienen lugar durante la estancia en la maternidad. Se considera que ocurre un error de medicación cuando se produce un incidente evitable, secundario a la utilización inapropiada de medicamentos que causa o puede causar un daño al paciente. ${ }^{5}$ Se presenta el caso de un neonato que recibió una dosis de enoxaparina diez veces superior a la terapéutica de forma accidental.

\section{CASO CLÍNICO}

Se trató de un recién nacido a término de bajo peso ( $2670 \mathrm{~g}$ ) que, a las $48 \mathrm{~h}$ de vida, recibió, por error, una dosis de $40 \mathrm{mg}$ de enoxaparina intramuscular (no subcutánea) destinada a una paciente (distinta de su madre), en lugar de la vacuna anti-hepatitis $B$. Ante el riesgo elevado de sangrado, se decidió el ingreso en la Unidad de Cuidados Intensivos Neonatales (UCIN) y se comunicó el error a los padres. Tras contactar con el Servicio de Hematología del centro y con la revisión bibliográfica previa, se concluyó que las guías consultadas recomendaban la administración de la misma dosis de sulfato de protamina como antídoto (40 $\mathrm{mg}$ en dosis única), aunque este medicamento no revertía 
completamente el efecto de las $\mathrm{HBPM},{ }^{6}$ tenía un margen terapéutico muy estrecho y no estaba exento de posibles efectos secundarios graves (hemorragias, inestabilidad hemodinámica, reacción anafiláctica, prolongación del QT y fenómenos trombóticos, entre otros). ${ }^{7}$ Por ello, se acordó su administración de forma fraccionada, orientada a la consecución de unos valores de factor anti-Xa $\leq 2 \mathrm{U} / \mathrm{ml}$, tal y como habían realizado otros autores. ${ }^{8}$ En la analítica inicial realizada (3,5 horas desde el evento), se observó un tiempo de tromboplastina parcial activado (TTPA) de 143,3 segundos, un tiempo de trombina $>120$ segundos con anti-Xa de 2,96 U/ml, y se pautó, en ese momento, una primera y única dosis de $15 \mathrm{mg}$ de sulfato de protamina. En las horas siguientes, se evidenció un descenso del anti-Xa, por lo que se decidió no administrar nuevas dosis (véase la tabla 1). Clínicamente, no se observaron signos de sangrado (petequias o hematomas). Se realizaron controles ecográficos tanto a nivel transfontanelar como abdominal el día del ingreso y en el momento del alta, que se llevaron a cabo tras 48 h en observación. Desde el punto de vista analítico, únicamente, se apreció un leve ascenso de gamma glutamil transpeptidasa (GGT) de $95 \mathrm{U} / \mathrm{L}$ en el primer control analítico realizado.

\section{COMENTARIO}

Existe una bibliografía muy limitada sobre el manejo de la sobredosis por enoxaparina en el período neonatal, y no se observa ningún caso descrito en castellano. Los errores cometidos con este tipo de medicación pueden suponer una amenaza real para la vida de los pacientes. Algunos autores, ante una situación similar (administración de $40 \mathrm{mg}$ de enoxaparina a un neonato a término), decidieron no administrar el antídoto, a pesar de tener unos valores máximos de anti-Xa de 3,16 U/ml y TTPA de 132 segundos a las 5,7 horas del evento, sin observar alteraciones en el paciente, aunque, en este caso, la presencia de un cefalohematoma al nacer secundario a la aplicación de ventosa pudo condicionar dicha actitud. En este caso, también observaron el aumento de GGT de hasta $341 \mathrm{U} / 1 .{ }^{9}$ Sin embargo, en un paciente pretérmino de 26 semanas con una edad corregida a término que estaba recibiendo tratamiento con enoxaparina para el manejo de una trombosis de la vena cava superior, otros autores ${ }^{8}$ optaron por la administración fraccionada de sulfato de protamina $(10,15$ y $10 \mathrm{mg}$ a la $1,45,4,5$ y 7,5 horas del evento, respectivamente), con un valor de anti-Xa $\leq 2 \mathrm{U} / \mathrm{ml}$ como objetivo. Teniendo en cuenta que el valor de anti-Xa para la monitorización de la enoxaparina en la población pediátrica es extrapolado de estudios realizados en adultos, con valores recomendados de 0,5-1 U/ $\mathrm{ml}$ en caso del empleo con fines terapéuticos y de $0,1-0,3 \mathrm{U} / \mathrm{ml}$ cuando su uso es profiláctico, ${ }^{10}$ en nuestro estudio, también se consideró un valor $\leq 2 \mathrm{U} / \mathrm{ml}$ adecuado en casos de sobredosificación, más aun cuando existían pocos estudios pediátricos que evaluaran la correlación entre la dosis de enoxaparina, los valores de anti-Xa y su eficacia clínica o la aparición de efectos adversos. De ahí que se optara por tratar de una forma intermedia, diferente a las publicadas, y se pautara una única dosis de sulfato de protamina, sin continuar con su administración una vez que los valores de anti-Xa hubieran alcanzado el valor perseguido y teniendo en cuenta la vida media de la enoxaparina. De esta forma, se evitó también la aparición de posibles efectos adversos relacionados con la administración del antídoto.

Este es el segundo caso descrito de sobredosificación de enoxaparina en recién

TABla 1. Valores de anti-Xa, tiempo de tromboplastina parcial activado y tiempo de trombina en función del tiempo desde el evento (administración de enoxaparina). Se indica el momento en el que se administró el sulfato de protamina

\begin{tabular}{lcccc}
\hline $\begin{array}{l}\text { Tiempo desde } \\
\text { el evento (horas) }\end{array}$ & $\begin{array}{c}\text { Actividad } \\
\text { anti-Xa (U/ml) }\end{array}$ & TTPA (s) & $\begin{array}{c}\text { Tiempo de } \\
\text { trombina (s) }\end{array}$ & $\begin{array}{c}\text { Administración de } \\
\text { sulfato de protamina }\end{array}$ \\
\hline 3,5 & 2,96 & 143,3 & $>120$ & Sí (15 mg) \\
5 & 2 & 60,6 & - & No \\
8 & 2 & 79,7 & $>120$ & No \\
13,5 & 1,1 & - & 22,9 & No \\
26 & 0,22 & - & 13,9 & No \\
48 & 0,1 & 39,4 & - & \\
\hline
\end{tabular}

Los valores no reflejados indican que la muestra no fue suficiente para su obtención. TTPA: tiempo de tromboplastina parcial activado. 
nacidos por su administración en vez de la vacuna anti-hepatitis $\mathrm{B}^{9}$ y el primero en castellano, lo que indica que casos similares, potencialmente graves, se pueden seguir repitiendo en nuestras unidades si no se establecen pautas de prevención apropiadas.

Existen diversas estrategias para la prevención de los errores en la administración de medicamentos, tales como la formación adecuada del personal, el empleo de protocolos escritos, las presentaciones farmacéuticas adaptadas al período neonatal, la participación de las autoridades sanitarias y de las compañías farmacéuticas, la prescripción electrónica y la monitorización continua de acontecimientos adversos, entre otras. ${ }^{11,12}$ En nuestro caso, las medidas de mejora establecidas en la planta de maternidad tras este hecho fueron la utilización de un carro exclusivo para la medicación neonatal, diferente al de las madres, y la prohibición de entrada en la habitación de medicación no perteneciente a los pacientes ingresados en ella.

\section{REFERENCIAS}

1. Nowak-Göttl U, Kosch A, Schlegel N. Thromboembolism in newborns, infants and children. Thromb Haemost. 2001;86(1):464-74.

2. $\mathrm{HoSH}, \mathrm{WuJK}$, HamiltonDP, etal. Anassessmentof published pediatric dosage guidelines for enoxaparin: a retrospective review. J Pediatr Hematol Oncol. 2004;26(9):561-6.

3. Leung M, Ho SH, Hamilton DP, et al. Utility of anti-Xa monitoring in children receiving enoxaparin for therapeutic anticoagulation. J Pediatr Pharmacol Ther. 2005;10(1):43-50.

4. EsquéRuiz MT, Moretones SuñolMG, Rodríguez Miguélez JM, et al. Los errores de tratamiento en una unidad neonatal, uno de los principales acontecimientos adversos. An Pediatr (Barc). 2016;84(4):211-7.

5. National Coordinating Council for Medication Error Reporting and Prevention. NCC MERP Taxonomy of medications errors. [Consulta: 28 de enero de 2018]. Disponible en: http://www.nccmerp.org/taxonomymedication-errors-now-available.

6. Crowther MA, Berry LR, Monagle PT, Chan AK. Mechanisms responsible for the failure of protamine to inactivate low-molecular-weight heparin. $\mathrm{Br}$ J Haematol. 2002;116(1):178-86.

7. España. Ministerio de Sanidad, Política Social e Igualdad, Agencia española de medicamentos y productos sanitarios. Ficha técnica Protamina. [Consulta: 2 de agosto de 2018]. Disponible en: https:/ / www.aemps.gob.es/cima/pdfs / es / ft / 45777/45777_ft.pdf.

8. Wiernikowski JT, Chan A, Lo G. Reversal of anti-thrombin activity using protamine sulfate. Experience in a neonate with a 10-fold overdose of enoxaparin. Thromb Res. 2007;120(2):303-5.

9. Mateos MK, WrightFA, Cohn RJ. Pharmacokinetic analysis of enoxaparin in a term neonate and review of literature. Thromb Res. 2013;132(4):487-9.

10. Monagle P, Chan A, Goldenberg NA, et al. Antithrombotic Therapy in neonates and children: Antithrombotic Therapy and Prevention of Thrombosis, $9^{\text {th }}$ ed: American College of Chest Physicians Evidence-Based Clinical Practice Guidelines. Chest. 2012;141(2 Suppl): e737s-801.

11. Ortega MJ, Domínguez Gil A. Acontecimientos adversos por medicamentos: una patología emergente. Farm Hosp. 2000;24(4):258-66.

12. Otero P, Leyton A, Mariani G, et al. Medication errors in pediatric inpatients: prevalence and results of a prevention program. Pediatrics. 2008;122(3):e737-43. 